\title{
Efficient and accurate calculation of exact exchange and RPA correlation energies in the Adiabatic-Connection Fluctuation-Dissipation theory
}

\author{
Huy-Viet Nguyen ${ }^{1,2}$ and Stefano de Gironcoli ${ }^{1,3}$ \\ ${ }^{1}$ SISSA-Scuola Internazionale Superiore di Studi Avanzati, via Beirut 2-4, I-34014 Trieste, Italy \\ ${ }^{2}$ Physics Faculty, Hanoi National University of Education, 136 Xuan-Thuy, Cau-Giay, Hanoi, Vietnam \\ ${ }^{3}$ CNR-INFM DEMOCRITOS National Simulation Centre, via Beirut 2-4, I-34014 Trieste, Italy
}

(Dated: April 26, 2022)

\begin{abstract}
Recently there has been a renewed interest in the calculation of exact-exchange and RPA correlation energies for realistic systems. These quantities are main ingredients of the so-called EXX/RPA+ scheme which has been shown to be a promising alternative approach to the standard LDA/GGA DFT for weakly bound systems where LDA and GGA perform poorly. In this paper, we present an efficient approach to compute the RPA correlation energy in the framework of the AdiabaticConnection Fluctuation-Dissipation formalism. The method is based on the calculation of a relatively small number of eigenmodes of RPA dielectric matrix, efficiently computed by iterative density response calculations in the framework of Density Functional Perturbation Theory. We will also discuss a careful treatment of the integrable divergence in the exact-exchange energy calculation which alleviates the problem of its slow convergence with respect to Brillouin zone sampling. As an illustration of the method, we show the results of applications to bulk $\mathrm{Si}$, Be dimer and atomic systems.

PACS numbers:
\end{abstract}

\section{INTRODUCTION}

Developments during the last several decades have brought Density Functional Theory (DFT) to be the method-of-choice in many calculations of physical and chemical properties from first-principles. Despite its many spectacular successes, Kohn-Sham (KS) densityfunctional theory within the LDA and GGAs approximations for the exchange-correlation (xc) functional still has some well-known drawbacks. This includes the failure of standard DFT functionals in the description of van der Waals systems where long-range correlation effects are predominant. This failure manifests clearly in erratic or completely failed results when LDA/GGA DFT is applied to sparse matter, including layered structures, polymer and molecular crystals, or weakly-bound chemical systems.

Of all the attempts to overcome these drawbacks, the approach based on the formally exact expression of xc energy in term of linear response functions derived from the Adiabatic Connection Fluctuation-Dissipation (ACFD) theorem 1 provides a promising way to develop a systematic improvement.

The method is in principle entirely parameter freealthough an appropriate definition of the xc kernel is still needed-and a few difficult cases where standard DFT fails qualitatively have been described with satisfactory results $2,3,5$ Practical applications in a few casestudies have shown that not only the description of van der Waals systems is improved but that the already satisfactory description of many molecules and solids are also not compromised. 3,4 The method is very computationally demanding and this has prevented its direct application to complex systems. Most often it is limited to a post-self-consistent correction where the accu- rate exchange-correlation energy is computed from the charge density obtained from a self-consistent calculation performed with a more traditional xc functional. The ACFD formalism also serves as the starting point for further simplifications that have led to the development of an approximate vdW-DF functional by Langreth and coworkers, $\frac{6}{-}$ which allows for the treatment of large systems and has recently been made fully self-consistent 7

We will address here the computational difficulties involved in the full evaluation of ACFD exchangecorrelation energies of realistic systems, presenting an efficient general formulation of the problem and its practical implementation in the plane-wave pseudopotential (PW PP) formalism.

Previous implementations of ACFD formulas rely on the construction of the full Kohn-Sham response function from the spectrum of the KS Hamiltonian. Basic definitions and some details are given in Sec. II] A more efficient scheme is then presented in Sec. III that avoids the full diagonalization of the KS Hamiltonian and the cumbersome summation over occupied and unoccupied states needed in the traditional calculation of the response functions, as well as the computationally demanding operations involved in the solution of the Dyson equation via exact algebra. These steps are replaced by the iterative calculation of a relatively small number of eigenmodes of the RPA dielectric matrix as explained in its general formulation in the first part of this section. Some technical details of the implementation in the PW PP formalism and a careful treatment of the integrable divergence which alleviates the problem of slow convergence with respect to Brillouin zone sampling in the calculation of the exact-exchange energy are also discussed here. To demonstrate the efficiency of the implementation, we will present in Sec. IV results of applications to selected 
paradigmatic systems, namely bulk $\mathrm{Si}$, weakly bound $\mathrm{Be}$ dimer, and a number of spherical atomic systems.

\section{THEORY}

We recall here some basic definitions in ACFD theory as well as some computational details of currently available PW PP implementations.

\section{A. Exchange and correlation energy in ACFD theory}

In the ACFD formalism, exchange-correlation energy, $E_{x c}$, of an electronic system with density $n(\mathbf{r})$ can be written in the formally exact form

$$
\begin{aligned}
E_{x c}= & -\frac{\hbar}{2 \pi} \int_{0}^{1} d \lambda \int d \mathbf{r} d \mathbf{r}^{\prime} \frac{e^{2}}{\left|\mathbf{r}-\mathbf{r}^{\prime}\right|} \\
& \times\left\{\int_{0}^{\infty} d u \chi_{\lambda}\left(\mathbf{r}, \mathbf{r}^{\prime} ; i u\right)+\delta\left(\mathbf{r}-\mathbf{r}^{\prime}\right) n(\mathbf{r})\right\},
\end{aligned}
$$

where $\chi_{\lambda}\left(\mathbf{r}, \mathbf{r}^{\prime} ; i u\right)$ is the density response function at imaginary frequency, $i u$, of the system where Coulomb interaction between electrons is scaled by $\lambda$, i.e. $\lambda e^{2} /\left|\mathbf{r}-\mathbf{r}^{\prime}\right|$, and the external potential is modified so that the electronic density is the same as in the ground state of the physical system $(\lambda=1)$. The exchange-correlation energy can be furthermore separated into the KS exchange energy, $E_{x}$, and the correlation energy, $E_{c}$. The former is the counterpart of Hartree-Fock exchange energy in the context of density-functional theory. It is given by the well-known expression in term of occupied KS orbitals (a non magnetic system is considered for simplicity and a factor of two accounts for spin degeneracy)

$$
E_{x}=-2 \frac{e^{2}}{2} \int d \mathbf{r} d \mathbf{r}^{\prime} \frac{\left|\sum_{i}^{o c c} \phi_{i}^{*}(\mathbf{r}) \phi_{i}\left(\mathbf{r}^{\prime}\right)\right|^{2}}{\left|\mathbf{r}-\mathbf{r}^{\prime}\right|} .
$$

The latter can be expressed in a compact form in terms of linear density responses, in matrix notation, as

$$
E_{c}=-\frac{\hbar}{2 \pi} \int_{0}^{1} d \lambda \int_{0}^{\infty} d u \operatorname{Tr}\left\{v_{c}\left[\chi_{\lambda}(i u)-\chi_{0}(i u)\right]\right\}
$$

In Eq. (33), $v_{c}$ is the electron-electron interaction kernel, $e^{2} /\left|\mathbf{r}-\mathbf{r}^{\prime}\right|$, and $\chi_{0}(i u)$ is the density response function of the non-interacting electron system and can be explicitly expressed in term of, occupied and empty, KS orbitals, $\phi_{i}(\mathbf{r})$, KS eigenvalues, $\epsilon_{i}$, and occupation numbers, $f_{i}$,

$$
\chi_{0}\left(\mathbf{r}, \mathbf{r}^{\prime} ; i u\right)=2 \sum_{i, j}\left(f_{i}-f_{j}\right) \frac{\phi_{i}^{*}(\mathbf{r}) \phi_{j}(\mathbf{r}) \phi_{j}^{*}\left(\mathbf{r}^{\prime}\right) \phi_{i}\left(\mathbf{r}^{\prime}\right)}{\epsilon_{i}-\epsilon_{j}+i \hbar u},
$$

where again a factor of two is present accounting for spin degeneracy. For $\lambda>0$, the interacting response function, $\chi_{\lambda}$, is related to $\chi_{0}$ by a Dyson-like equation with an exchange-correlation kernel, $f_{\lambda}^{x c}(i u)$, which is still unknown:

$$
\chi_{\lambda}=\chi_{0}+\chi_{0}\left[\lambda v_{c}+f_{\lambda}^{x c}\right] \chi_{\lambda} .
$$

In the above formula, frequency dependence is implied in each term (with the exception of the Coulomb kernel, $v_{c}$ ) and spatial coordinate dependence is implicit in the matrix notation. Despite the exactness of the expressions presented above, one needs to use an approximation for the xc-kernel in practical applications. When the xckernel is specified, the system of Eqs. (3) - (5) is closed and allows one to evaluate the correlation energy.

\section{B. Total energy in EXX/RPA + scheme}

Experience has shown that none of the available approximate xc-kernels gives a systematic improvement; different kernels perform better for different systems. Although the RPA-kernel, i.e. simply setting $f_{\lambda}^{x c}$ in Eq. (5) to zero, is exact for long-range correlation, it is a poor approximation for short-range correlation. When applied to atomic or molecular systems, RPA correlation energy alone is very inaccurate. In fact, it was abandoned decades ago as a DFT approximate xc functional until a quite recent work by Kurth and Perdew $\stackrel{\underline{8}}{\underline{2}}$ showed that it is possible to correct the deficiency of RPA in a simple way by combining the full RPA with a local- or semilocaldensity correction for short-range correlation. This leads to the so-called RPA+ correlation energy in which the local-density correction for the short-range correlation is chosen in such a way that $E_{\mathrm{c}}^{\mathrm{RPA}+}$ becomes exact in the limit of homogeneous electron gas (HEG)

$$
E_{\mathrm{c}}^{\mathrm{RPA}+}=E_{\mathrm{c}}^{\mathrm{RPA}}-\left(E_{\mathrm{c}}^{\mathrm{LDA}-\mathrm{RPA}}-E_{\mathrm{c}}^{\mathrm{LDA}}\right) .
$$

In the above definition, $E_{\mathrm{c}}^{\mathrm{LDA}-\mathrm{RPA}}$ is the local-density approximation of RPA correlation energy which is exactly canceled out by $E_{\mathrm{c}}^{\mathrm{RPA}}$ in the limit of an HEG.

In order to have exchange and correlation energies treated on the same footing, exchange energy must also be computed in the same formalism, i.e. from Eq. (2).

In practice this EXX/RPA+ scheme is applied as a post-scf correction and the energy is calculated by first performing a standard LDA/GGA DFT calculation and then replacing the xc-energy at LDA level by exactexchange and RPA + correlation energies calculated from the LDA/GGA charge density. In spite of this, the EXX/RPA + scheme has been shown to be a promising approach for the description of weakly bound systems where standard LDA/GGA performs poorly $: \underline{2,3,5}$

\section{Existing implementations in PW PP method}

Existing implementations $\frac{9}{}$ of the ACFD formulas in plane-wave pseudopotential computer codes start by first diagonalizing the KS Hamiltonian for all the occupied 
and unoccupied KS orbitals,,$\frac{5}{5}$ or at least a good part of the unoccupied states, $\stackrel{2,3}{=}$ so that the KS response function $\chi_{0}(i u)$ can be calculated explicitly according to its definition in Eq. (4). The calculation then proceeds by solving the Dyson-like equation for $\chi_{\lambda}(i u)$ for a number of values of the coupling constant, $\lambda$, and of the imaginary frequency, $i u$, and then by integrating over these variables to ultimately obtain the correlation energy. An obvious disadvantage of these implementations is that many unoccupied states need to be considered in order to get well-converged results. This forces one to solve the KS problem using full matrix diagonalization algorithms with unfavorable scaling instead of very efficient iterative-diagonalization techniques, commonly used to calculate the occupied KS orbitals in typical selfconsistent calculations. Moreover the summation over valence and conduction bands and over all the k-points in the Brillouin zone for setting up the response function (Eq. (4)) has been shown to be a very cumbersome operation which prevents the application of the method to large systems.

\section{EFFICIENT CALCULATION OF ACFD FORMULAS}

We describe now our method, first focusing on its general aspects, valid for any symmetry and basis set, and then specializing the discussion to a PW PP approach.

\section{A. General aspects and PW PP implementation}

Let us starts by defining the following generalized eigenvalue problems

$$
\begin{aligned}
& \chi_{0}\left|w_{i}\right\rangle=a_{i} v_{c}^{-1}\left|w_{i}\right\rangle, \\
& \chi_{\lambda}\left|z_{i}^{\lambda}\right\rangle=b_{i}^{\lambda} v_{c}^{-1}\left|z_{i}^{\lambda}\right\rangle,
\end{aligned}
$$

where $\chi_{0}, \chi_{\lambda}$ and $v_{c}$ are linear response and Coulomb operators in matrix notation, $\left\{\left|w_{i}\right\rangle, a_{i}\right\}$ and $\left\{\left|z_{i}^{\lambda}\right\rangle, b_{i}^{\lambda}\right\}$ are eigenpairs (all these quantities depend implicitly on the imaginary frequency $i u$ ). These eigenvalues problems are well-defined since all the operators are symmetric and positive $\left(v_{c}\right)$ or negative $\left(\chi_{0}, \chi_{\lambda}\right)$ definite. Once solutions of the generalized eigenvalue problems are available, the traces of $v_{c} \chi_{\lambda}$ and $v_{c} \chi_{0}$ in Eq. (3) are simply evaluated by summing up all their (relevant) eigenvalues. Working with these generalized eigenvalue problems has several advantages when RPA approximation is considered: (i) the set of formally $\lambda$-dependent eigenpotentials, $\left\{\left|z_{i}^{\lambda}\right\rangle\right\}$, is actually $\lambda$-independent and coincides with its non-interacting counterpart, $\left\{\left|w_{i}\right\rangle\right\}$, (ii) the corresponding eigenvalues are trivially related as

$$
b_{i}^{\lambda}=\frac{a_{i}}{1-\lambda a_{i}}
$$

which (iii) allows one to perform the $\lambda$ coupling constant integration in Eq. (3) analytically leading to the final expression

$$
E_{c}=\frac{\hbar}{2 \pi} \int_{0}^{\infty} d u \sum_{i}\left\{a_{i}(i u)+\ln \left(1-a_{i}(i u)\right)\right\} .
$$

The spectrum of the eigenvalue problem in Eq. (7) is bounded from above by zero, which is also an eigenvalue, corresponding to a constant eigenpotential. Note that the eigenvalues $\left\{a_{i}\right\}$ are closely related to those of the RPA dielectric matrix since $\epsilon_{R P A}=1-v_{c} \chi_{0}$, and the calculation of $\left\{a_{i}\right\}$ is very similar to the calculation of dielectric band structures introduced a few decades ago $\stackrel{10,11}{=}$ In principle, the full spectra must be known in order to calculate the correlation energy from Eq. (10) above. In practice, however, previous calculations $10,11,12$ have shown that only a small number of eigenvalues of the RPA dielectric matrix significantly differs from unit. This translates in the fact that only a small fraction of the eigenvalues $\left\{a_{i}\right\}$ will be significantly different from zero and needs to be explicitly included in the correlation energy sum; all the rest being so close to zero that their contributions to $E_{c}$ can be treated in a suitable approximation or even simply neglected.

Evaluation of low-lying eigenvalues of the noninteracting problem in Eq. (77) is done efficiently by iterative diagonalization procedure starting from a set of trial eigenpotentials. The basic operation involved in the iterative diagonalization is the calculation of the noninteracting response to a trial potential, $\Delta n=\chi_{0} \Delta v$. This is done resorting to the linear response techniques of Density Functional Perturbation Theory $\stackrel{13}{\leftrightarrows}$ simply generalized to imaginary frequency.

Technically, for any given non-interacting perturtbing potential, $\Delta v$, the induced charge density variation is

$$
\Delta n(\mathbf{r})=2 \operatorname{Re} \sum_{i}^{o c c} \phi_{i}^{*}(\mathbf{r}) \Delta \phi_{i}(\mathbf{r})
$$

where the sum runs over the occupied (valence) states and $\Delta \phi_{i}(\mathbf{r})$ is the (conduction-band projected) variation of the single-particle state, $\phi_{i}(\mathbf{r})$, that can be obtained as the solution of the linear system:

$$
\left[H_{K S}+\alpha P_{v}-\left(\epsilon_{i}+i u\right)\right]\left|\Delta \phi_{i}\right\rangle=-\left(1-P_{v}\right) \Delta v\left|\phi_{i}\right\rangle,
$$

where $P_{v}=\sum_{i}^{o c c}\left|\phi_{i}\right\rangle\left\langle\phi_{i}\right|$ is the projector on the occupied manifold and $\alpha$ is a positive constant, larger than the valence bandwidth, ensuring that the linear system is not singular even in the limit for $i u \rightarrow 0$. The nonhermitian linear systems are solved by employing a fast and smoothly converging variant of the bi-conjugate gradient algorithm $\underline{15}$

Overall the procedure amounts to solving the usual DFPT linear systems $\underline{13}$ in which the ground state valence eigenvalues are shifted by the complex constant $i u$, as already explained by Mahan 14 long time ago for the case of atomic polarizabilities. Note however that, at variance with standard DFPT, no self-consistent cycle is needed to 
obtain $\Delta n$ as the response of the non-interacting system is being considered here.

As in static DFPT calculations, only occupied KohnSham orbitals and their linear response need to be calculated, instead of the full KS spectrum needed in other implementation $\mathbf{2}^{2,3,5}$

The scheme describe above have been implemented in the Quantum ESPRESSO distribution.16 Very similar ideas have also been recently reported by Wilson and $\operatorname{coworkers}^{17}$ for the calculation of dominant eigenpotentials of static dielectric matrices.

The description presented so far is to a large extent basis set independent. For periodic systems, matrix representations of the Kohn-Sham linear response functions satisfy the Bloch theorem and can be classified by a vector $\mathbf{q}$ in the Brillouin zone. The generalized eigenvalue problem in Eq. (7) can therefore be solved separately for each $\mathbf{q}$ vector. As a consequence, the expression for the correlation energy per unit cell becomes

$E_{c}=\frac{\hbar}{2 \pi} \int_{0}^{\infty} d u \frac{1}{N_{q}} \sum_{q=1}^{N_{q}} \sum_{i}^{N_{e i g}}\left\{a_{i}(\mathbf{q}, i u)+\ln \left(1-a_{i}(\mathbf{q}, i u)\right)\right\}$

where the notation $\frac{1}{N_{q}} \sum_{q=1}^{N_{q}}$ indicates the average over the Brillouin zone and can be performed using the special point technique that takes advantage of the point group symmetry of the system.

Special care is required in the limit of $\mathbf{q} \rightarrow 0$ since the leading matrix elements $\chi_{0}^{00}(\mathbf{q} \rightarrow 0)$ and $v_{c}^{-1}$ both go to zero as $|\mathbf{q}|^{2}$ making the eigenvalue problem (7) ill-defined. A simple solution to the problem is to use shifted grids of $\mathbf{q}$ vectors in the special-point summation.

\section{B. Exchange energy}

Although the main focus of this work is the calculation of correlation energy it is clear that an accurate evaluation of the exchange term is also necessary. A subtle problem, not widely recognized in the literature, related to the $\mathbf{q} \rightarrow 0$ divergence of the Coulomb interaction is present in the calculation of exact-exchange energy in a plane-wave approach and special care must be payed to it.
The exchange energy per unit cell for a periodic system is defined as

$$
E_{x}=-\frac{e^{2}}{N} \sum_{\substack{\mathbf{k} v \\ \mathbf{k}^{\prime} v^{\prime}}} \int \frac{\phi_{\mathbf{k} v}^{*}(\mathbf{r}) \phi_{\mathbf{k}^{\prime} v^{\prime}}(\mathbf{r}) \phi_{\mathbf{k}^{\prime} v^{\prime}}^{*}\left(\mathbf{r}^{\prime}\right) \phi_{\mathbf{k} v}\left(\mathbf{r}^{\prime}\right)}{\left|\mathbf{r}-\mathbf{r}^{\prime}\right|} d \mathbf{r} d \mathbf{r}^{\prime}
$$

where an insulating and non magnetic system is assumed for simplicity. Integrals and wave-function normalizations are defined over the whole crystal volume, $V=N \Omega$ ( $\Omega$ being the unit cell volume), and the summations run over all occupied bands and all $\mathrm{N}$ k-points defined in the BZ by Born-von Karman boundary conditions.

Introducing codensities, $\rho_{\substack{\mathbf{k}^{\prime}, v^{\prime} \\ \mathbf{k}, v}}(\mathbf{r})=\phi_{\mathbf{k}^{\prime}, v^{\prime}}^{*}(\mathbf{r}) \phi_{\mathbf{k}, v}(\mathbf{r})$, with Fourier transform, $\rho_{\mathbf{k}, v} \mathbf{k}^{\prime}, v^{\prime}\left(\mathbf{k}-\mathbf{k}^{\prime}+\mathbf{G}\right)$, the above expression can be recast in reciprocal space as

$$
E_{x}=-\frac{4 \pi e^{2}}{\Omega} \times \frac{\Omega}{(2 \pi)^{3}} \int d \mathbf{q} \sum_{\mathbf{G}} \frac{A(\mathbf{q}+\mathbf{G})}{|\mathbf{q}+\mathbf{G}|^{2}}
$$

where an auxiliary regular function $A(\mathbf{q}+\mathbf{G})=$ $\frac{1}{N} \sum_{\mathbf{k}} \sum_{v, v^{\prime}}\left|\rho_{\mathbf{k}, v}^{\mathbf{k}-\mathbf{q}, v^{\prime}}(\mathbf{q}+\mathbf{G})\right|^{2}$ has been introduced.

A direct integration of Eq. (15) on the regular q-point grid, defined by the $\mathbf{k}-\mathbf{k}^{\prime}$ differences of the points used for the wave-function BZ sampling, is problematic due to integrable divergence that appears in the $\mathbf{q}+\mathbf{G} \rightarrow 0$ limit. The problem has been addressed several time in the literature and many related schemes have been proposed to treat it. Most eventually resort to a procedure, first proposed by Gygi and Baldereschi, 18 where an easily integrable term, that displays the same divergence, is subtracted from and separately added back to Eq. (15). Of the many proposed forms $\frac{18,19,20}{}$ we adopt here one of the simplest that can be straightforwardly applied to any crystal structure: we simply subtract and add a term $A(0) e^{-\alpha|\mathbf{q}+\mathbf{G}|^{2}} /|\mathbf{q}+\mathbf{G}|^{2}$ from the integrand. The integration of the resulting non divergent term can then be performed by a summation over a regular grid of points (either the full q-point grid defined from the $\mathbf{k}$-point differences or, in order to reduce computational cost, on a subgrid of it), while the divergent integration can be performed explicitly.

The final results is

$$
E_{x}=-\left\{\frac{4 \pi e^{2}}{N_{\mathbf{q}} \Omega}\left[\sum_{\mathbf{q}, \mathbf{G}} \frac{A(\mathbf{q}+\mathbf{G})}{|\mathbf{q}+\mathbf{G}|^{2}}+\left\langle\left\langle\lim _{\mathbf{q} \rightarrow 0} \frac{A(\mathbf{q})-A(0)}{\mathbf{q}^{2}}\right\rangle\right\rangle\right]+D A(0)\right\}
$$

with

$$
D=\frac{4 \pi e^{2}}{N_{\mathbf{q}} \Omega}\left[-\sum_{\mathbf{q}, \mathbf{G}} \frac{e^{-\alpha|\mathbf{q}+\mathbf{G}|^{2}}}{|\mathbf{q}+\mathbf{G}|^{2}}+\alpha\right]+\frac{4 \pi e^{2}}{(2 \pi)^{3}} \sqrt{\frac{\pi}{\alpha}} .
$$


The symbol $\sum^{\prime}$ in the preceding formulas implies that the term $\mathbf{q}+\mathbf{G}=0$ is excluded. Notice that with our simple choice the expression for the divergence correction, $D$, is just (the reciprocal-space part of) the Ewald sum for a single point-charge, periodically repeated according to the super-periodicity defined by the q-point grid used. As long as the parameter $\alpha$ is chosen so that the reciprocal space sum is converged the result is independent of $\alpha$ and gives a correction that decay very slowly with the inverse of the cell linear dimension, $L$. This $1 / L$ dependence of the singularity correction is for instance evident in Fig. 2 of Ref. 20, although the scaling law of the correction was not explicitly pointed out there. In general, the error incurred on by neglecting this correction would be so large that it is almost invariably included in the calculation.

From a closer analysis of Eq. (16), however, it is also clear that, even when the main effect of the divergence has been taken into account by the GygiBaldereschi procedure, another correction needs to be included to properly describe the spherically-averaged limit, $\left\langle\left\langle\lim _{\mathbf{q} \rightarrow 0} \frac{A(\mathbf{q})-A(0)}{\mathbf{q}^{2}}\right\rangle\right\rangle$, that cannot be calculated di- rectly for $\mathbf{q}=0$.

It can be shown, see the Appendix, that this term is simply related to the gauge invariant spread of the occupied manifold, as can be defined in the theory of maximally localized Wannier functions $\underline{\underline{22}}$ If this term is neglected (as to the best of our understanding it has always been neglected so far in the literature) an error proportional to this spread and inversely proportional to the cell volume and the number of $\mathbf{q}$-points included in the $\mathrm{BZ}$ summation is made. This might be the reason for the reported slow convergence of the exchange energy with respect to BZ integration, even for simple systems such as bulk Silicon $\underline{\underline{4}}$ or Argon, $\underline{\underline{5}}$

We have therefore included in the calculation of the exchange energy an estimate of the limiting term, based on the assumption that the grid of q-points used for BZ integration is dense enough that a coarser grid, including only every second point in each direction would also be equally accurate. Since the limiting term contribute to the integral with different weight in the two grids one can estimate its value as:

$$
\left\langle\left\langle\lim _{\mathbf{q} \rightarrow 0} \frac{A(\mathbf{q})-A(0)}{\mathbf{q}^{2}}\right\rangle\right\rangle=\frac{1}{7} \sum_{\text {dense }}{ }^{\prime} \frac{A(\mathbf{q}+\mathbf{G})}{|\mathbf{q}+\mathbf{G}|^{2}}-\frac{8}{7} \sum_{\text {coarse }} \frac{{ }^{\prime}}{|\mathbf{q}+\mathbf{q}+\mathbf{G}|^{2}} .
$$

We have verified that the convergence with respect to BZ integration is generally improved. For instance for the exchange energy of solid Argon, calculated from LDA wave-functions, a regular grid of $6 \times 6 \times 6$ points was sufficient for a convergence of the order of $0.1 \mathrm{mRy}$, while a much denser $12 \times 12 \times 12$ grid was needed in Ref. 5 .

\section{Computational cost}

To discuss the computational cost of our implementation, let us denote by $N_{v}$, and $N_{c}$ the number of valence and conduction bands, and by $N_{p w \psi}$, and $N_{p w \chi_{0}}$ the number of plane waves used to represent wave functions and Kohn-Sham response functions, respectively. The basic operations in our implementation is the calculation of the linear density response via DFPT technique. For a norm-conserving pseudopotential, the computational cost of a linear response calculation is essentially $N_{v} \times\left(N_{v} \times N_{p w \psi}+N_{p w \chi_{0}}\right)$. Since this calculation is done repeatedly in the iterative diagonalization procedure, the total computational cost must be multiplied by the number of eigenvalues $\left(N_{\text {eig }}\right)$ that we want to calculate and the number of iterations $N_{i t e r}$ in the iterative diagonalization. While the latter is likely independent of the system size, it is expected and in fact there is evidence $\frac{17}{\underline{7}}$ that the former is proportional to it.
Therefore the total scaling of our approach is proportional to $N_{p w \psi} N_{v}^{2} N_{e i g}$ which grows as the fourth power of the system size. In other implementations of ACFD formulas which are based on the evaluation of the full response matrix, the most time-consuming operation is the construction of the non-interacting Kohn-Sham response function whose computational cost is proportional to $N_{p w \chi_{0}}^{2} N_{v} N_{c}$. This means that the computational cost of these approaches also scales with the fourth power of the system size.

Note however that $N_{p w \psi}$ is typically smaller than $N_{p w \chi_{0}}$ by an order of magnitude since $\chi_{0}$ relates to density responses and perturbing potentials whose kineticenergy cutoff is four times larger than the one needed for wave-functions in the case of norm conserving pseudopotentials and even more than that in the case of ultra-soft pseudopotentials. Also the number of eigenpotentials that needs to be computed is expected to be at least an order of magnitude smaller than the size of the response function $\left(N_{p w \chi_{0}}\right)$ and the number of iteration $N_{\text {iter }}$ is unlikely to exceed 10, especially if in the imaginary frequency scan good starting trial eigenpotentials are taken from the previous frequency in the list. Our rough estimate shows therefore that the number of operations involved in our method is from 100 to 1000 smaller than the one needed for other implementations. Although real CPU-time obviously depends on many de- 
tails in the realization of each approach, we are confident that our approach allows us to implement ACFD formulas in a very efficient way.

Moreover, our approach also has significant advantages due to its iterative nature. Iterative solution of the generalized eigenvalue problem (8) will converge very rapidly if the initial guess of the eigenpotentials are already close to the sought solutions. As mentioned earlier calculated solutions for a given imaginary frequency can serve as starting points for nearby frequencies. A similar behavior can be expected also for calculation of response functions in nearby $\mathbf{q}$-vectors although it may be more convenient in this case to exploit the very easy parallelization of the q-vector summation involved in the correlation energy formula, Eq. (13). Finally, as already mentioned in the general formulation, the analysis of the response function in terms of eigenmodes allows us to analytically perform the coupling constant integration with a significant computational saving.

\section{APPLICATION TO SELECTED SYSTEMS}

\section{A. Bulk Si}

We have chosen bulk Si system as a testing ground for our implementation of ACFD theory since the computational cost for the case of bulk $\mathrm{Si}$ is rather moderate, which is convenient for convergence checks, and there exist several published data that we can compare with. As a first check, we have calculated the 20 topmost eigenvalues of the dielectric matrix at $\mathbf{q}=(0,0,0.01) \frac{2 \pi}{0}$ and compared our results with those reported in Ref. 17 where the same quantities were calculated by the explicit diagonalization of the RPA dielectric matrix and by an iterative diagonalization procedure, named Projective Dielectric EigenPotential method (PDEP), similar to ours 23 The results reported in Table $\amalg$ show perfect agreement of the two methods among each other and with the explicit calculation.

Let us now investigate the convergence of RPA correlation energy with respect to the number of eigenmodes included in the summation of Eq. (13). To this purpose, we have used well-converged parameters for standard LDA calculation of silicon ground-state charge density to evaluate the exact-exchange and RPA-correlation energies. The calculation was performed for the diamond structure in the fundamental face-centered cubic cell with lattice constant of 10.20 Bohr (corresponding to the theoretical equilibrium geometry at LDA level) using a regular grid of $64 \mathrm{k}$-point and a kinetic-energy cut-off of 20 Rydberg.

We show in Fig. 1 the dependence of RPA correlation energy, $E_{c}^{R P A}$, on the number of eigenvalues, $N_{e i g}$, included in the summation. $E_{c}^{R P A}$ is indeed a rapidly converging function of $N_{\text {eig }}$; truncating the sum after inclusion of 80 or 100 eigenvalues already ensures a convergence within a few tens of mRy. Also the summation over special q-points representing the integration in the

\begin{tabular}{crrr}
\hline \hline Index & $\mathrm{RPA}^{17}$ & $\mathrm{PDEP}^{17}$ & present work \\
\hline 1 & 14.7432 & 14.7538 & 14.7611 \\
2 & 3.4231 & 3.4237 & 3.4238 \\
3 & 3.3908 & 3.3914 & 3.3915 \\
4 & 3.3908 & 3.3914 & 3.3915 \\
5 & 3.3908 & 3.3914 & 3.3915 \\
6 & 3.3908 & 3.3914 & 3.3915 \\
7 & 3.3589 & 3.3596 & 3.3596 \\
8 & 2.4910 & 2.4925 & 2.4925 \\
9 & 2.4910 & 2.4925 & 2.4925 \\
10 & 2.4910 & 2.4925 & 2.4925 \\
11 & 2.4910 & 2.4925 & 2.4925 \\
12 & 2.4905 & 2.4920 & 2.4920 \\
13 & 2.4905 & 2.4920 & 2.4920 \\
14 & 2.4716 & 2.4721 & 2.4721 \\
15 & 2.1964 & 2.1972 & 2.1972 \\
16 & 2.1960 & 2.1968 & 2.1968 \\
17 & 2.1959 & 2.1966 & 2.1967 \\
18 & 2.1959 & 2.1966 & 2.1967 \\
19 & 2.1958 & 2.1966 & 2.1967 \\
20 & 2.1958 & 2.1966 & 2.1967 \\
\hline \hline
\end{tabular}

TABLE I: Top 20 eigenvaules of the dielectric matrix for an eight-atom silicon cubic cell calculated via the present iterative method and compared with he results reported in Ref. 17 for RPA and for projective dielectric eigenpotential method

first Brillouin zone converges very rapidly; the correlation energy changes only by a few mRy when the number of special points increases from 2 to 6 .

Next we compare our accurately calculated exchange and $\mathrm{RPA}(+)$ correlation energies with the energies calculated within the pseudopotential approximation but using quantum Monte Carlo techniques as reported by Hood and co-workers in Ref. 24. Table I shows clearly

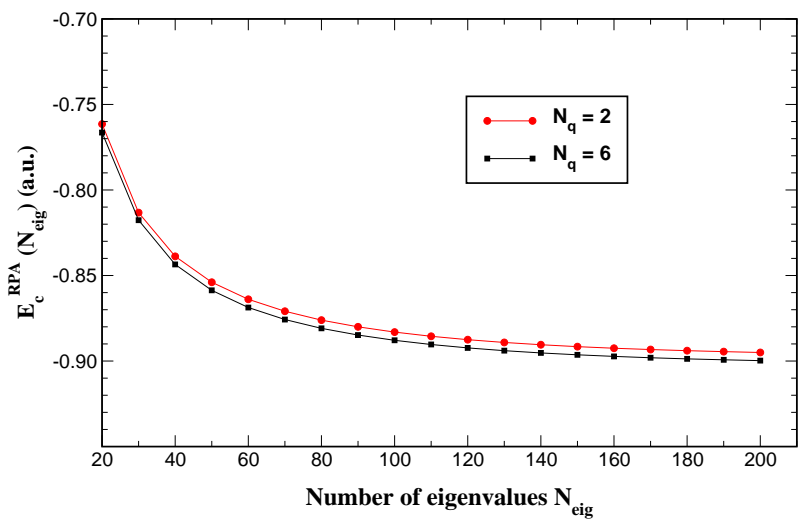

FIG. 1: RPA correlation energy as function of the number of eigenvalues included in the summation in Eq. (13) for fcc bulk silicon. The two curves are for different number of special $\mathbf{q}$ points in the Brillouin zone integration of the same equation. 


\begin{tabular}{cccc}
\hline \hline & $a_{0}(\mathrm{bohr})$ & $E_{x}(\mathrm{eV})$ & $E_{c}(\mathrm{eV})$ \\
\hline $\mathrm{VMC}$ & -- & -29.15 & $-3.58 \pm 0.01$ \\
$\mathrm{DMC}$ & -- & -29.15 & $-4.08 \pm 0.08$ \\
& & & \\
$\mathrm{EXX} / \mathrm{RPA}$ & 10.20 & -29.11 & -6.12 \\
& 10.26 & -28.98 & -6.11 \\
$\mathrm{EXX} / \mathrm{RPA}+$ & 10.20 & -29.11 & -4.24 \\
& 10.26 & -28.98 & -4.23 \\
\hline \hline
\end{tabular}

TABLE II: Exchange and correlation energies for bulk Si calculated by our method compared to the values calculated using QMC techniques by Hood and co-workers reported in Ref. 24.

that RPA alone gives indeed too negative values for the correlation energy. When a simple local-density correction is added (RPA+), the presently calculated correlation energy becomes much closer to the Monte Carlo values. Obviously, the comparison would be more convincing had we used the same pseudopotential used by Hood and co-workers. Nonetheless, we believe that the good agreement between our calculations and the results obtained by a very different method shows that our results are at least in the right regime.

We have also calculated the total energy of bulk silicon as a function of its lattice constant in EXX/RPA+ scheme in order to determine the corresponding equi-

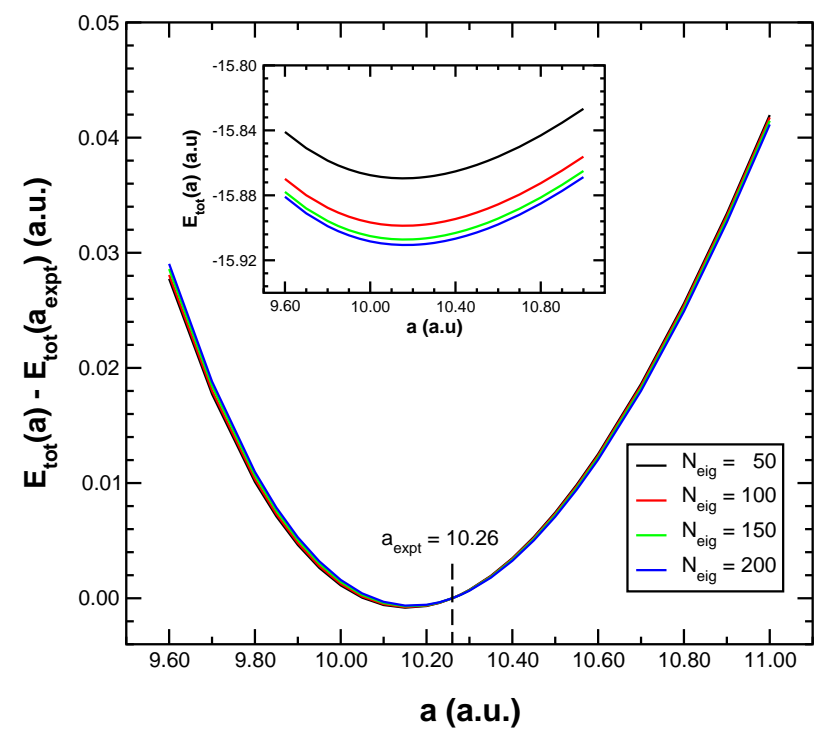

FIG. 2: The total energy (per unit cell) differences at different lattice constants and that at the experimental one of bulk silicon calculated using different number of eigenvalues values included in the summation in Eq. (10) in our implementation of EXX/RPA+ scheme. The inset is the true total energy. i.e. the values at the experimental lattice constant is not subtracted. librium properties. Fig. 2 shows the total energy per unit cell for bulk silicon as a function of lattice constant calculated including different numbers $N_{e i g}\left(N_{e i g}=\right.$ $50,100,150$ and 200) of response function eigenvalues in the summation in Eq. (10). For each curve the value calculated at the experimental lattice constant is subtracted. The resulting (nearly) coincidence of the different curves confirms the expectation that energy differences are rather insensitive to the number of eigenvalues included in the RPA sum. This can also be appreciated in the inset, where the unshifted EXX/RPA+ total energies are shown: as more eigenvalues are included in the sum energy vs volume curves rigidly shift down and although complete convergence is reached only when including about 200 eigenvalues already 100 or even 50 eigenvalues basically return the same structural properties.

Table III collect the predicted equilibrium lattice parameter $a_{0}$, bulk modulus $B$ and pressure derivative of the bulk modulus $B^{\prime}$ as a function of the number of eigenvalues $N_{e i g}$ used to evaluate RPA correlation energies. The changes in these quantities when increasing $N_{\text {eig }}$ from 50 to 200 is very small, of the order of $0.1 \%$ for $a_{0}$ and $B$ and $1 \%$ for $B^{\prime}$. We can therefore conclude that only a relatively small number of eigenvalues are needed in the evaluation of ACFD RPA correlation energy to get a good description of many equilibrium properties of the system.

Examining the predicted equilibrium lattice constants in Table III it seems that a more accurate (and sophisticated) treatment of xc-energy in EXX/RPA+ scheme slightly worsens the agreement with experimental data as compared with the LDA results. Nevertheless, as already pointed out in Ref. 4, there are several points that can affect the final results: (i) EXX/RPA+ scheme is applied in a non self-consistent way using LDA Kohn-Sham orbitals, (ii) the pseudopotential itself has been generated within LDA, and (iii) RPA+ is among the simplest possible approximations to the xc-kernel within ACFD formalism. In view of these shortcoming we believe that the

\begin{tabular}{|c|c|c|c|c|}
\hline$N_{e i g}$ & & $a_{0}$ (a.u.) & $B(\mathrm{GPa})$ & $B^{\prime}$ \\
\hline 50 & & 10.155 & 99.5 & 4.22 \\
\hline 100 & & 10.158 & 99.4 & 4.21 \\
\hline 150 & & 10.162 & 99.3 & 4.19 \\
\hline \multirow[t]{3}{*}{200} & & 10.166 & 99.1 & 4.17 \\
\hline & LDA & 10.235 & 92.5 & 4.16 \\
\hline & $\operatorname{Expt}^{a}$ & 10.26 & 99.2 & 4.15 \\
\hline
\end{tabular}

${ }^{a}$ Experimental values are taken from Ref. 25

TABLE III: Predicted equilibrium lattice parameter, $a_{0}$, bulk modulus, $B$, and pressure derivative of the bulk modulus, $B^{\prime}$, as function of the number of eigenvalues $N_{\text {eig }}$ used to evaluate RPA+ correlation energies. The corresponding LDA and experimental values are also shown for comparison. 
present results for Silicon can be considered satisfactory.

In spite of the improvement of numerical efficiency in our implementation, we also observed a slow convergence of RPA correlation energy with respect to the kineticenergy cutoff as reported in other implementations. 3,5 Efficient extrapolation schemes that allow for the evaluation of correlation energies in the limit of infinite energy cutoff, already proposed for those implementations, can be easily adapted to ours. As for the convergence with respect to BZ sampling, we notice, as explicitly shown in Ref. 26 for the case of bulk Si, that the difference between RPA correlation energies calculated using different number of k-points (for charge density) and q-points (for $E_{c}$ ) is a well-behaved function of the kinetic-energy cutoff, beyond a certain (not very large) value. This suggests an extrapolation scheme, similar to the one proposed in Ref. 3, that might become useful for the calculation of more complex systems for which even the present very efficient implementation would be too demanding to reach complete convergence directly. The extrapolation procedure would be as follows: First a coarse grid of $\mathbf{k}$ - and q-point could be used for the calculation of RPA correlation energies at different kinetic-energy cutoffs. Second, the corresponding correlation energy in the infinite cutoff limit could be obtained by extrapolating the results obtained at finite cutoffs. Finally, the errors due to coarse $\mathbf{k}$ - and $\mathbf{q}$-point sampling of the BZ could be corrected by using finer grids evaluated at small kinetic-energy cutoff (whose safe value could be estimated from the convergence behavior of the correlation energy computed with the coarser grids.)

\section{B. $\mathrm{Be}_{2}$ dimer}

Beryllium dimer is a paradigmatic example of the failure of LDA/GGA DFT in the description of weakly bound systems. Previous studies 2,27 have shown considerable discrepancies between LDA/GGA and experimental results for binding energy, bond length and vibrational frequencies. While the errors of LDA/GGA bond lengths are in fact quite small (less than $2 \%$ ), the vibrational frequency is largely overestimated. The most severe discrepancy refers to the binding energy: both LDA and GGA approximations overestimate the experimental value by at least a factor of 4 . Fuchs and Gonze ${ }^{2}$ have recently shown that $\mathrm{EXX} / \mathrm{RPA}+$ improves significantly the description of $\mathrm{Be}_{2}$ dimer over standard DFT. However, the error-bar of the RPA correlation energy reported in that study is still as large as $30 \mathrm{meV}$ which is of the same order of the experimental binding energy and the reported theoretical value. Our efficient implementation of ACFD formulas allows us to reach a better congergence, and thus to provide a better assessment of the performance of EXX/RPA + formalism for this system. To this end, we have carefully checked the convergence with respect to all parameters involved in our calculations, namely the kinetic-energy cutoff, the size

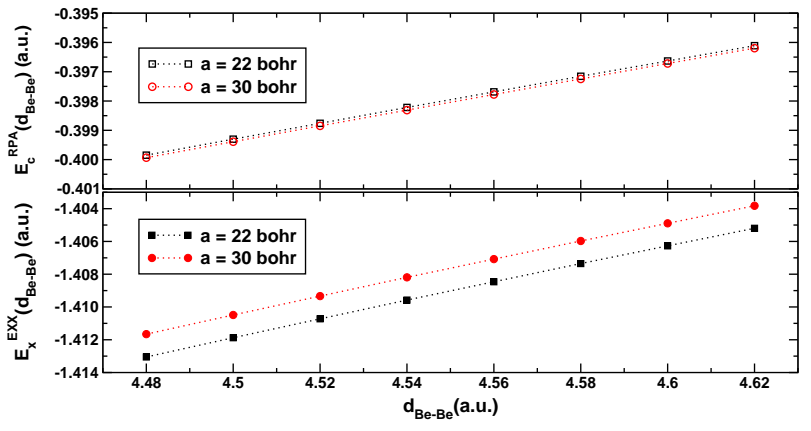

FIG. 3: Exact-exchange and RPA correlation energies of $\mathrm{Be}_{2}$ as a function of Be-Be distance $\left(d_{B e-B e}\right)$ as calculated in a simple cubic cell of side length of 22 (square) and 30 (circle) Bohr. Dotted lines are simply drawn as a guide to the eye.

of the supercell used to simulate an isolated system using a periodic plane-wave approach, and the number of eigenvalues included in the evaluation of RPA correlation energy.

The same norm-conserving pseudopotential used in Ref. 2, generated within exact-exchange Kohn-Sham formalism, has been used here. It is a rather soft pseudopotential and increasing the plane-wave kinetic-energy cutoff from 20 to 30 Ry makes the LDA total energy, the exact-exchange and RPA correlation energies change less than $0.5 \mathrm{mRy}$. This good convergence is consistent with the use in Ref. 2 of a plane-wave kinetic-energy cutoff of $25 \mathrm{Ry}$.

Convergence with respect to the size of supercell is more delicate. Figure 3 shows exact-exchange and RPA correlation energies as a function of Be-Be distances calculated using supercells of 22 and 30 Bohr. While RPA correlation energy varies by only a fraction of a mRy (top panel), the lower panel shows a significant variation of several $\mathrm{mRy}$ for the exact-exchange energy. As discussed in Sec. IIIB slow convergence of exact-exchange energy with respect to supercell size-or the density of the BZ sampling for an extended system-can result if, once the integrable divergence is eliminated by the GygiBaldereschi procedure, $\stackrel{18}{\stackrel{1}{1}}$ the residual $\mathbf{q}=0$ term is not estimated correctly. This is shown in Figure 4 where the exact exchange energy of $\mathrm{Be}_{2}$ molecule is shown as a function of the inverse supercell volume. A large error, proportional to the inverse supercell volume, is present when the residual $\mathbf{q}=0$ term is simply neglected, while a much better convergence with system size is obtained when it is estimated according to the recipe described in Sec. IIIB. While with a simple cubic supercell of 22 Bohr side the calculated exchange energy still has an error of a couple of $m R y$, we are fully confident that using a supercell of 30 Bohr ensures a convergence of the exactexchange energy within a fraction of mRy. One might expect some degree of error cancellation in energy differences when the residual $\mathbf{q}=0$ terms are neglected in the exact-exchange calculations of Be dimer and of the separated Be atoms. Our explicit verification has shown 


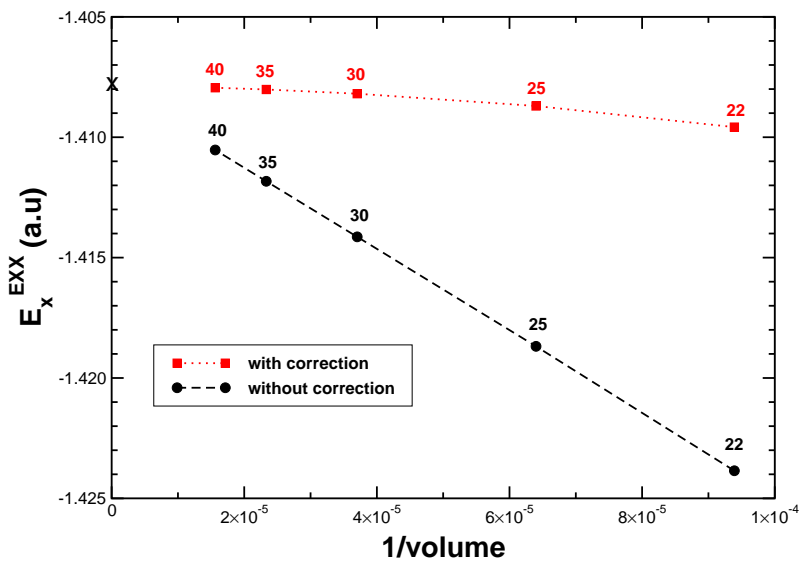

FIG. 4: Exchange energy of $\mathrm{Be}_{2}$ as a function of the supercell volume. Both the calculation neglecting (filled circles) and including (filled squares) an estimate of the $\mathbf{q}=0$ term in the sum are reported. Numbers close to the symbols correspond to the supercell linear dimension in Bohr.

that a variation of the order of $m R y$ is still present in this difference when the supercell size is increased from 22 to 30 bohr.

Finally, convergence of RPA correlation energy with respect to the number of eigenvalues included in the ACFD

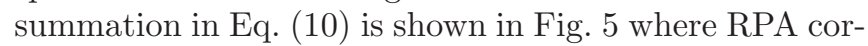
relation energies of $\mathrm{Be}_{2}$ calculated using 120, 180, and 220 eigenvalues are plotted as a function of Be-Be distances. By including up to 220 eigenvalues, a convergence within $0.5 \mathrm{mRy}$ is obtained for the absolute value of correlation energy. On the basis of the nearly parallel behavior of the curves reported in Fig. 5 for different number of included eigenvalues we can anticipate a much faster convergence for energy differences, which actually determine the equilibrium properties of the dimer.

Table IV collects predicted binding energy, bond length, and vibrational frequency of $\mathrm{Be}_{2}$ calculated including different numbers of eigenvalues in the ACFD evaluation of RPA correlation energy. As expected, all these quantities are only very slightly changed when the

\begin{tabular}{ccccccccc}
\hline \hline \multirow{2}{*}{$N_{\text {eig }}$} & \multicolumn{2}{c}{$\mathrm{E}_{b}(\mathrm{eV})$} & & \multicolumn{2}{c}{$\mathrm{d}_{0}(\mathrm{bohr})$} & & \multicolumn{2}{c}{$\omega_{e}\left(\mathrm{~cm}^{-1}\right)$} \\
\cline { 2 - 3 } & $\mathrm{RPA}$ & $\mathrm{RPA}+$ & & $\mathrm{RPA}$ & $\mathrm{RPA}+$ & $\mathrm{RPA}$ & $\mathrm{RPA}+$ \\
\hline 60 & -0.0667 & -0.0377 & & 4.516 & 4.553 & & 296.1 & 298.5 \\
120 & -0.0657 & -0.0368 & & 4.521 & 4.558 & & 296.6 & 298.7 \\
180 & -0.0655 & -0.0367 & & 4.523 & 4.560 & & 296.3 & 299.2 \\
220 & -0.0654 & -0.0365 & & 4.524 & 4.561 & & 297.1 & 298.5 \\
& & & & & & & & \\
Ref. & $-0.08(3)$ & $-0.06(3)$ & & 4.55 & 4.59 & & 311 & 298 \\
Expt. & \multicolumn{2}{c}{-0.098} & & \multicolumn{2}{c}{4.63} & & 275.8 \\
\hline \hline
\end{tabular}

TABLE IV: Equilibrium properties of $\mathrm{Be}_{2}$ in $\operatorname{EXX} / \mathrm{RPA}(+)$ scheme with different numbers of eigenvalues included in the calculation of RPA correlation energy.

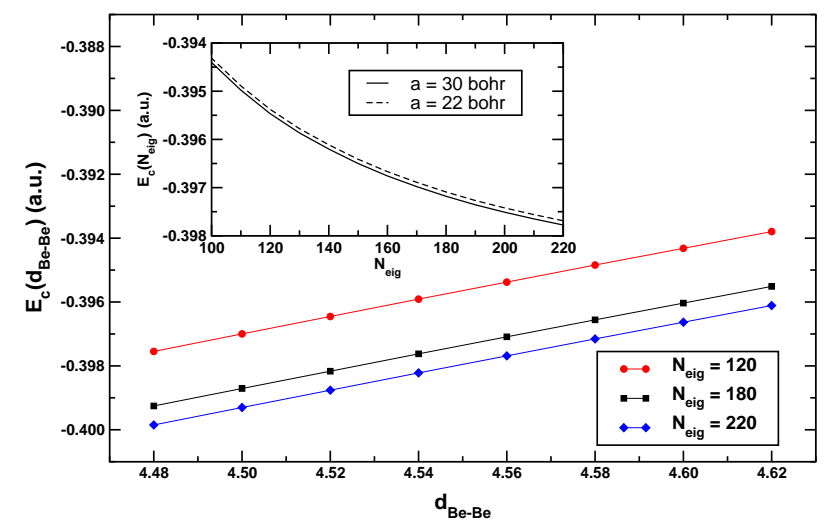

FIG. 5: RPA correlation energies of $\mathrm{Be}_{2}$ at different Be-Be distances $\left(d_{B e-B e}\right)$. The curves are for different number of eigenvalues $N_{\text {eig }}$ included for evaluation of Eq. (10): $N=120$ (circle), $N=180$ (square), and $N=220$ (diamond). The lines are simply drawn as a guide. Inset: RPA correlation energies of $\mathrm{Be}_{2}$ at the $\mathrm{Be}-\mathrm{Be}$ distance $\left(d_{B e-B e}\right)$ of 4.56 Bohr placed in a simple cubic supercell with the size length of 22 (dashed line) and 30 Bohr (solid line).

number of eigenvalues is increased from 60 to a value as large as 220. Even the molecular binding energy appears to have a convergence of the order of the $\mathrm{meV}$ when only 60 eigenvalues are included in the ACDF sum. The result that properties related to energy differences are rather insensitive to the number of eigenpotentials used in evaluating RPA correlation energy, not only in the "standard" case of silicon (section IVA), but also in the much more delicate case of $\mathrm{Be}_{2}$ dimer is very promising for the future application of the present method to complex realistic systems.

When compared with the reference values reported in Ref. 2, our binding energies, both in RPA and RPA+, are systematically smaller, although still in agreement within the large error bar of the previous calculation. Moreover, had we calculated the binding energy of $\mathrm{Be}_{2}$ without the $\mathbf{q}=0$ correction described above, as done in the earlier calculation, 28 a much better matching with the ACFD calculation in Ref. 2 would have been obtained.

Coming to the comparison with the experimental results it is clear that while EXX/RPA+ scheme definitely improves the poor performance of LDA and GGA for weakly bound systems like $\mathrm{Be}_{2}$, the results may not be as good as suggested in Ref. 22. The performance of RPA+ and other ACFD-based schemes to describe realistic weakly bound systems needs to be more systematically investigated.

\section{Spherical atomic systems}

Benchmark results of RPA correlation energies for a number of spherical atoms calculated by constructing the full response function from the spectrum of Kohn-Sham Hamiltonian have been recently reported in Ref. 32 . 
TABLE V: Full RPA and RPA+ correlation energy (in Rydberg atomic units) of spherical atoms compared to the reference and exact values. The reference data were calculated from EXX-only (i.e. exact-exchange and no correlation) KS orbitals in a different implementation ${ }^{32}$

\begin{tabular}{|c|c|c|c|c|c|c|c|c|}
\hline \multirow[t]{2}{*}{ Atom } & \multirow[t]{2}{*}{$E_{c}^{\text {expt }}$} & \multirow[t]{2}{*}{$E_{c}^{\mathrm{LDA}}$} & \multicolumn{3}{|c|}{$E_{c}^{\mathrm{RPA}}$} & \multicolumn{3}{|c|}{$E_{c}^{\mathrm{RPA}+}$} \\
\hline & & & $\rho^{\mathrm{LDA}}$ & $\rho^{\mathrm{EXX}}$ & Ref. 32 & $\rho^{\mathrm{LDA}}$ & $\rho^{\mathrm{EXX}}$ & Ref. 32 \\
\hline $\mathrm{He}$ & $-0.084^{a}$ & -0.229 & -0.168 & -0.167 & -0.166 & -0.096 & -0.094 & 0.094 \\
\hline $\mathrm{Be}$ & $-0.190^{a}$ & -0.447 & -0.373 & -0.367 & -0.358 & -0.230 & -0.224 & 0.216 \\
\hline $\mathrm{Ne}$ & $-0.786^{a}$ & -1.474 & -1.216 & -1.195 & -1.194 & -0.821 & -0.800 & 0.800 \\
\hline $\mathrm{Ar}$ & $-1.463^{a}$ & -2.842 & -2.221 & -2.206 & -2.202 & -1.503 & -1.487 & 1.482 \\
\hline $\mathrm{Kr}$ & $-4.15^{b}$ & -6.533 & -5.226 & -5.192 & $\mathrm{n} / \mathrm{a}$ & -3.736 & -3.702 & $\mathrm{n} / \mathrm{a}$ \\
\hline $\mathrm{Xe}$ & $-6.86^{b}$ & -10.358 & -8.312 & -8.278 & $\mathrm{n} / \mathrm{a}$ & -6.049 & -6.016 & $\mathrm{n} / \mathrm{a}$ \\
\hline
\end{tabular}

a Experimental values quoted in Ref. 8.

$b$ Difference of "exact" and HF total energies reported in Ref. 31 .

For spherically symmetric systems, ground state KohnSham orbitals are classified by their principal quantum number, $n$, and by angular momentum numbers $l, m$. The KS equations can be solved numerically on a radial grid, within a given approximate, LDA or GGA, functional. Similarly, the non interacting and interacting response functions are block-diagonal with respect to angular momentum $l$, and $(2 l+1)$-fold degenerate with respect to $m$. Thus, contributions to the ACFD formula from different angular momenta can be calculated independently and added up. For each angular momentum the calculation proceeds as follows. (i) A trial potential is selected and the corresponding linear density response is calculated by solving the modified Sternheimer equation. $\stackrel{14}{=}$ A single iteration, with no self-consistency, is needed since the non-interacting response-function is studied. (ii) The calculated density response is orthogonalized, with overlap matrix $v_{c}$, with respect to any previously computed one. The generating potential is accordingly transformed, exploiting the linearity of $\chi_{0}$. By cal-

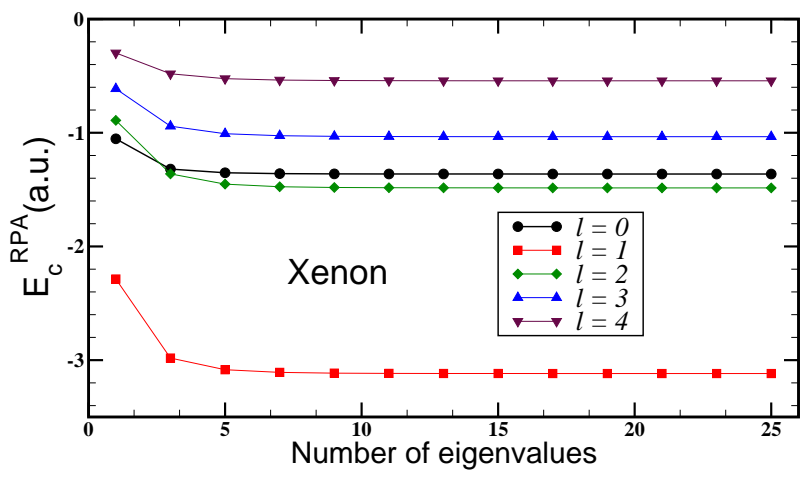

FIG. 6: The dependence of RPA correlation energy on the number of eigenvalues for Xenon atom. The curves show the cumulative contribution to RPA correlation energy for some of the lowest angular momentum numbers (higher $l$ are not plotted). Including the first 15 eigenvalues is enough to ensure a convergence within $1 m R y$, which is also used as the threshold for convergence with respect to angular number $l$. culating the Hartree potential from the resulting density a new trial perturbation is generated. (iii) A matrix representation of $\chi_{0}$ on the hence generated trial-potential basis is built, and diagonalized to get the eigenvalues $a_{i}$ 's. This three-step process is repeated until convergence in the sum over eigenvalues in Eq. (10) is reached. The same calculation procedure is then repeated at different values of angular momentum, $l$, and imaginary frequency, $i u \stackrel{29}{2}$

Let us investigate the convergence of the RPA $E_{c}$ with respect to the number of eigenvalues of the generalized eigenvalue problem of Eq. (77). Fig. 6] shows the dependence of RPA correlation energy-separated in the different angular momentum contributions-on the number of eigenvalues included in the sum for Xenon atom, the heaviest atom considered in this work (56 electrons). Basis set convergence is carefully checked and, for the case of Xe, a basis-set size of 25 trial potentials is enough for the desired accuracy. It is clearly seen that the correlation energy converges quite rapidly; including up to 15 eigenvalues is enough in order to convergence the total correlation energy within $1 m R y$. These calculations therefore confirm explicitly, also for the case of spherical atoms, our expectation that RPA correlation energy can be obtained from only a small number of eigenmodes of the non-interacting response-function of the system.

Table V shows the full RPA and RPA+ correlation energies calculated with our method for a number of spherical atoms whose ground state densities have been generated from EXX-only $\stackrel{30}{\underline{3}}$ and standard LDA functionals. Experimental $\underline{\underline{8}}$ or accurate theoretical ${ }^{31}$ values are also shown for comparison, together with reference RPA and

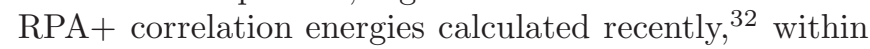
a different implementation of the ACFD approach, from EXX-only charge densities. All our calculated values in table $\mathbf{V}$ are converged within a few mRy. The results slightly depend on the quality of the ground-state density, i.e. on the different approximate xc-functionals used in the self-consistent ground state calculation. Focusing on the results obtained starting from EXX-only charge densities, our calculated values for the full RPA correla- 
tion energy (the fifth column) agree well with the reference data (sixth column) within the error bar (with the exception of Be case); a similar agreement is found for $\mathrm{RPA}+$ correlation energies (eighth and ninth columns of table V). The small residual differences between the values obtained in the present and in the reference calculations may probably be attributed to some slight residual difference in the electronic densities used as input.

Not surprisingly, RPA correlation energies alone largely overestimate the exact values. When combined with a local-density corrections to form the RPA+ approximation the correlation energy compare very favorably with the exact values thus giving support to the validity of RPA+ scheme.

\section{CONCLUSION}

In this work, we have proposed an efficient method for the calculation of RPA correlation energies in the adiabatic-coupling fluctuation-dissipation formalism. Our approach involves the evaluation of a relatively small number of eigenvalues of the non-interacting response function, obtained combining concepts from density functional perturbation theory with iterative diagonalization techniques. General strategies as well as technical details of the method as implemented in the plane-wave pseudopotential Quantum-ESPRESSO distribution have been discussed to some extent.

We have applied the method to study a few systems, representative of bulk solids, weakly bound molecules and atoms. While the study of bulk silicon crystal helps validate the implementation, our study of Beryllium dimer, thanks to its improved numerical accuracy with respect to previous studies, allows us to gain a clearer picture of the performance of EXX/RPA+ scheme in describing weakly bound systems. Our calculation confirms the important improvements of $\mathrm{EXX} / \mathrm{RPA}+$ with respect to LDA or GGA but also shows that its performance in delicate cases can be less impressive than previously concluded. The good agreement of RPA+ correlation energy with experimental or accurate theoretical results for a few spherical atoms lend anyhow support to the quality of RPA+ correlation energies.

We are confident that the possibility of a careful control of the numerical accuracy in ACFD calculation, resulting from the improved numerical efficiency of our method, will turn out to be very useful in the needed analysis of the performance of such a sophisticated den- sity functional in the study of many other realistic systems.

\section{Acknowledgments}

We thank M. Fuchs for providing us the pseudopotential used for calculations in Ref. 2 and for useful discussions.

\section{APPENDIX}

In this appendix we demonstrate that the sphericallyaveraged limit, $\left\langle\left\langle\lim _{\mathbf{q} \rightarrow 0} \frac{A(\mathbf{q})-A(0)}{\mathbf{q}^{2}}\right\rangle\right\rangle$, is simply related to the gauge invariant spread of the occupied KS manifold, $\Omega_{I}$, defined in the theory of maximally localized Wannier functions 22 as

$$
\Omega_{I}=\sum_{n}\left[\left\langle\mathbf{0} n\left|\mathbf{r}^{2}\right| \mathbf{0} n\right\rangle-\sum_{\mathbf{R}, m}|\langle\mathbf{0} n|\mathbf{r}| \mathbf{R} m\rangle|^{2}\right],
$$

where $\{|\mathbf{R} n\rangle\}$ is (any) set of Wannier functions that describes the occupied manifold.

Starting from the explicit expression for $A(\mathbf{q})$ in terms of the occupied Bloch states

$$
A(\mathbf{q})=\frac{1}{N} \sum_{\mathbf{k}} \sum_{v, v^{\prime}}\left\langle\phi_{\mathbf{k}, v}\left|e^{-i \mathbf{q} \mathbf{r}}\right| \phi_{\mathbf{k}-\mathbf{q}, v^{\prime}}\right\rangle\left\langle\phi_{\mathbf{k}-\mathbf{q}, v^{\prime}}\left|e^{+i \mathbf{q r}}\right| \phi_{\mathbf{k}, v}\right\rangle,
$$

let us expand them in terms of the Wannier functions,

$$
\left|\phi_{\mathbf{k}, v}\right\rangle=\sum_{n} U_{v, n}(\mathbf{k}) \sum_{\mathbf{R}} e^{i \mathbf{k R}}|\mathbf{R} n\rangle,
$$

where $U_{v, n}(\mathbf{k})$ are general unitary transformations in the set of occupied bands at point $\mathbf{k}$ in the BZ. Inserting this definition in the expression for $A(\mathbf{q})$, this becomes, after some straightforward manipulation,

$$
A(\mathbf{q})=\sum_{\mathbf{R}} \sum_{n, m}\left\langle\mathbf{R} n\left|e^{-i \mathbf{q r}}\right| \mathbf{0} m\right\rangle\left\langle\mathbf{0} m\left|e^{+i \mathbf{q r}}\right| \mathbf{R} n\right\rangle .
$$

Expanding now the exponential factors in powers of $\mathbf{q}$ and taking the spherically averaged limit for $\mathbf{q} \rightarrow 0$ one easily obtains the desired result:

$$
\left\langle\left\langle\lim _{\mathbf{q} \rightarrow 0} \frac{A(\mathbf{q})-A(0)}{\mathbf{q}^{2}}\right\rangle\right\rangle=-\frac{\Omega_{I}}{3} .
$$

1 D.C. Langreth and J.P. Perdew, Solid State Commun. 17, 1425 (1975); Phys. Rev. B 15, 2884 (1977).

2 M. Fuchs and X. Gonze, Phys. Rev. B 65, 235109 (2002).

3 A. Marini, P. García-González, and Angel Rubio, Phys. Rev. Lett. 96, 136404 (2006).

4 P. García-González, J. J. Fernández, A. Marini, and A.
Rubio, J. Phys. Chem. A. 111, 12458 (2007).

5 J. Harl and G. Kresse, Phys. Rev. B 77, 045136 (2008).

6 D. C. Langreth et al., J. Quant. Chem. 101, 599 (2005).

7 T. Thonhauser et al., Phys. Rev. B 76, 125112 (2007).

8 S. Kurth and J. P. Perdew, Phys. Rev. B 59, 10461 (1999).

${ }^{9}$ Note, however, that there exists also an implementation 
with unfavorable scaling based on atomic orbital basis as shown in F. Furche, Phys. Rev. B 64, 195120 (2001) and F. Furche and T. Van Voorhis, J. Chem. Phys. 122, 164106 (2005).

10 A. Baldereschi and E. Tosatti, Solid State Commun. 29, 131 (1979).

11 R. Car, E. Tosatti, S. Baroni, and S. Leelaprute, Phys. Rev. B 24, 985 (1981).

12 M. S. Hybertsen and S. G. Louie, Phys. Rev. B 35, 5585 (1987).

13 S. Baroni, S. de Gironcoli, A. Dal Corso, and P. Giannozzi, Rev. Mod. Phys. 73, 515 (2001).

14 G. D. Mahan, Phys. Rev. A 22, 1780 (1980).

15 H.A. van der Vorst, SIAM J. Sci. Stat. Comput. 13, 631664, (1992).

16 S. Baroni et al., http://www.quantum-espresso.org/. See also http://www.pwscf.org/.

17 H. F. Wilson, F. Gygi, and G. Galli, Phys. Rev. B 78, 113303 (2008).

18 F. Gygi, and A. Baldereschi, Phys. Rev. B 34, 4405 (1986).

19 J. Paier, R. Hirschl, M. Marsman, and G. Kresse, J. Chem. Phys. 122, 234102 (2005).

20 P. Carrier, S. Rohra, and A. Görling, Phys. Rev. B 75, 205126 (2007).

21 J. Spencer, and A. Alavi, Phys. Rev. B 77, 193110 (2008).

22 N. Marzari, and D. Vanderbilt, Phys. Rev. B 56, 12847 (1997).

${ }^{23}$ In the calculation by the PDEP method, the results were obtained from the diagonalization of the interacting response function $(\chi)$ instead of the noninteracting KohnSham one as in our implementation. Therefore, in order to obtain eigenvalues of the RPA dielectric matrix, the exchange-correlation part of the potential in DFPT formalism was disabled while solving DFPT equations selfconsistently.

${ }^{24}$ R. Q. Hood, M. Y. Chou, A. J. Williamson, G. Rajagopal, and R. J. Needs, Phys. Rev. B 57, 8972 (1998)

25 O. H. Nielsen and R. M. Martin, Phys. Rev. B 32, 3780 (1985).

26 More details can be found here: Huy-Viet Nguyen, PhD thesis, International School for Advanced Studies (SISSA), 2008, available at http://www.sissa.it/cm/phd.php

27 S. L. Richardson, M. Y. Chou, and M. L. Cohen, Phys. Rev. A 31, 3444 (1985).

28 M. Fuchs (private communication).

29 For an efficient integration over imaginary frequencies in Eq. (10), we divide the frequency axis into several intervals and integrate within each interval by the Gauss-Legendre method. Convergence within $1 m R y$ is ensured by going to frequency high enough that the absolute value of the integrand is as small as $10^{-8}$. Such a tight threshold is very critical, especially for heavy atoms, since frequencies as high as $10^{6}$ Ry must be considered and special care must be taken in order to reach this accuracy. This is necessary because the kernel in the correlation energy expression decays, roughly, as the inverse of the sum of the squares of the imaginary frequency and of the largest excitation energy in the system which is of the order of thousands Rydberg for heavy atoms like $\mathrm{Kr}$ and Xe. As for the summation over angular momentum components, including up to $l=20$ is typically enough to ensure the convergence within $1 m R y$.

30 A. Görling, and M. Levy, Phys. Rev. A 50, 196 (1994); R.J. Magyar, A. Fleszar, and E.K.U. Gross, Phys. Rev. B 69, 045111 (2004).

31 A. Ma, N. D. Drummond, M. D. Towler, and R. J. Needs, Phys. Rev. E 71, 066704 (2005). Strictly speaking these are correlation energies in HF sense, their numerical value should however be very close to the one for correlation energies in DFT.

32 H. Jiang and E. Engel, J. Chem. Phys. 127, 184108 (2007). 